This item is the archived peer-reviewed author-version of:

\title{
Interleukin-15 : new kid on the block for antitumor combination therapy
}

\section{Reference:}

Van den Bergh Johan, van Tendeloo Vigor, Smits Evelien.- Interleukin-15 : new kid on the block for antitumor combination therapy

Cytokine and growth factor reviews - ISSN 1359-6101 - 26:1(2015), p. 15-24

DOI: http://dx.doi.org/doi:10.1016/j.cytogfr.2014.09.001

Handle: http://hdl.handle.net/10067/1231210151162165141 


\title{
Interleukin-15: new kid on the block for antitumor combination therapy
}

\author{
Johan M.J. Van den Bergh ${ }^{1}$, Viggo F.I. $\underline{\text { Van Tendeloo }}{ }^{1}$, Evelien L.J.M. $\underline{\text { Smits }}^{1,2}$ \\ ${ }^{1}$ Laboratory of Experimental Hematology, Vaccine and Infectious Disease Institute (VAXINFECTIO), \\ Faculty of Medicine \& Health Sciences, University of Antwerp, Antwerp, Belgium \\ ${ }^{2}$ Center for Oncological Research Antwerp, Faculty of Medicine \& Health Sciences, University of \\ Antwerp, Antwerp, Belgium
}

Corresponding author: Smits ELM, Laboratory of Experimental Hematology, Antwerp University Hospital (UZA), Wilrijkstraat 10, B-2650 Edegem, Belgium.

Tel.: 003238214683; Fax.: 003238214456. E-mail address: evelien.smits@uza.be

\begin{abstract}
Interleukin (IL)-15 is one of the most promising molecules to be used in antitumor immune therapy, as it is able to stimulate the main killer cells of both the innate and adaptive immune system. Although this cytokine can be used as a stand-alone immunotherapeutic agent, IL-15 will probably be most efficient in combination with other strategies to overcome high tumor burden, immune suppression of the tumor microenvironment and/or the short half-life of IL-15. In this review, we will discuss the combination strategies with IL-15 that have been tested to date in different animal tumor models, which include chemotherapy, other immunostimulatory cytokines, targeted therapy, adoptive cell transfer and gene therapy. In addition, we give an overview of IL-15 combination therapies that are currently tested in clinical studies to treat patients with hematological or advanced solid tumors.
\end{abstract}

\section{Keywords}

Interleukin-15, combination therapy, tumor immunotherapy, natural killer cells, CD8 ${ }^{+} \mathrm{T}$ cells

\section{Abbreviations}

ALT-803, IL-15 + sushi domain of IL-15R $\alpha$; BMT, bone marrow transplantation; CTLA, cytotoxic Tlymphocyte antigen; CTVT, canine transmissible venereal tumors; DC, dendritic cells; EGFR, epidermal growth factor receptor; GVHD, graft-versus-host disease; GVT, graft-versus-tumor; IL, interleukin; IL-15R, IL-15 receptor; MDSC, myeloid-derived suppressor cells; moABs, monoclonal antibodies; NK, natural killer; PD1, programmed cell death 1; RFA, radiofrequency thermal ablation; rh, recombinant human; TGF, transforming growth factor; Th, T helper 


\section{Background}

Since its discovery two decades ago, interleukin (IL)-15 has become one of the most promising molecules for antitumor immunotherapy, illustrated by its top position in the US National Cancer Institute's ranking of 20 immunotherapeutic drugs with the greatest potential for broad usage in cancer therapy [1]. IL-15 owes its nomination to its ability to stimulate both the innate and the adaptive immune system. More specifically, IL-15 is a pivotal factor on one hand in the development, proliferation and activation of natural killer (NK) cells, and on the other hand in $\mathrm{CD}^{+} \mathrm{T}$-cell proliferation and activation [2,3]. On top, IL-15 is also a main player in the activation of NKT cells, $\gamma \delta T$ cells, B cells and increases the antibody-secreting capacity of the latter [4-6]. Due to its pleiotropic functions, IL-15 forms a bridge between both immune systems, which is in favour of optimal defense against malignant cells [7].

At its discovery, IL-15 was thought to exert similar functions as IL-2, because both cytokines share the $\beta$ - and common $\gamma$-chains of their receptor, which contains the cytoplasmic motifs required for signal transduction. Interestingly however, IL-2 and IL-15 display different functions in vivo, which may be due to the $\alpha$-moiety of the respective receptors [8-10]. In addition to activating $\mathrm{CD}^{+} \mathrm{T}$ cells, IL-2 causes activation-induced cell death and provokes maintenance of $\mathrm{CD} 4^{+} \mathrm{CD} 25^{+}$regulatory $\mathrm{T}$ cells, in contrast to IL-15 [11, 12]. These unique properties of IL-15 may be of benefit in the immunotherapy of cancer [8]. Indeed, IL-15-mediated immune stimulation occurs mainly by a unique trans-presentation mechanism, whereby IL-15 bound to the $\alpha$-moiety of the IL-15 receptor (IL-15R $\alpha$ ) is being trans-presented to the $\beta \gamma$-chains of its receptor on neighboring cells [13-15]. In addition, IL-15 can bind to the IL-15 $\beta$-receptor without forming a pre-complex with IL-15R $\alpha$, although with a lower binding affinity [16]. Moreover, pre-complexation of IL-15 with IL-15R $\alpha$ results in an increase of the IL-15 half-life $[17,18]$. The different binding strategies of IL-15 to its receptor subunits are depicted in Figure 1 . Since both NK cells and CD8 ${ }^{+} \mathrm{T}$ cells as the main killers of malignant cells express IL-15 $\beta \gamma$-receptor, IL-15 is considered to be an important mediator of antitumor immunity.

IL-15 has proven to possess immunostimulatory capacities both in vitro and in preclinical models. Therefore, IL-15 has recently been incorporated in three phase I clinical trials as a single agent to treat multiple types of advanced solid tumors (NCT01727076, NCT01021059, NCT01572493). Most likely, combining IL-15 with agents that work synergistically to activate immunity or deplete immune-regulating/suppressing cells may result in more vigorous immune responses and improved clinical outcome [19]. In this regard, IL-15 can be combined with strategies to (i) overcome high tumor burden, (ii) further strengthen the antitumor immune response and/or (iii) block inhibitory strategies which are used by tumors to promote their survival. In this review, we discuss the therapeutic combination strategies with IL-15 examined to date in animal tumor models. Furthermore, we summarize ongoing clinical trials that test recombinant human (rh)IL-15 as an (adjuvant) therapy in combination with other antitumor therapies. 


\section{Lessons from preclinical tumor models}

IL-15 as immunotherapeutic agent is an excellent adjuvant as observed in mouse, beagle and rat tumor models (Table 1). In these studies, IL-15 is combined with other antitumor strategies, namely (i) chemotherapeutic agents, (ii) immunosuppressors, (iii) other cytokines, (iv) DNA- or tumor vaccines ( $v$ ) immune-activating antibodies, (vi) inhibitory antibodies of immune-suppressive molecules or (vii) adoptive cell therapy.

\section{Chemotherapy}

Nowadays, first-line treatment of most cancers consist of surgery, chemotherapy, radiotherapy or a combination of these. Although in most cases the tumor burden is reduced significantly, chemotherapy/radiotherapy-resistant remnant tumor cells contribute to a high relapse rate of tumor patients. Therefore, adjuvant immunotherapy is currently intensively being investigated to improve treatment outcome of tumor patients by eliminating chemotherapy-resistant tumor cells using the patient's own immune system. Interestingly, some chemotherapeutic agents can stimulate a process called 'immunogenic cell death', in which tumor cells undergo apoptosis while emitting a combination of signals that can elicit a long-term protective antitumor immune response [20-22]. Five agents causing immunogenic cell death have been identified to date, including cyclophosphamide [23]. In addition, many other chemotherapeutics, such as 5-fluorouracil, can modulate the tumor's phenotype (e.g. upregulating carcinoembryonic antigen and downregulating $\mathrm{Bc} / 2$ expression), rendering them more sensitive to immune-mediated killing [24, 25]. In this context, IL-15 as a potent immunostimulatory molecule may act synergistically with chemotherapeutic agents by repairing or strengthening the antitumor functions of immune effectors that are involved in the patient's response to chemotherapy (Figure 2).

IL-15 has been tested in combination with (1) cyclophosphamide; and (2) 5-fluoruracil and leucovorin. The combination therapy with cyclophosphamide, an alkylating cytotoxic chemotherapeutic, and IL-15 resulted in a prolonged survival of both C57BL/6J mice bearing rhabdomyosarcoma [26] and established pulmonary metastases [27]. The prolonged survival following treatment with the combination therapy may be due to an increased percentage of $\mathrm{NK} 1.1^{+} / \mathrm{LGL}^{+}$cells and $\mathrm{CD}^{+} / \mathrm{CD}_{4} 4^{+}$memory $\mathrm{T}$ cells in the blood as compared with chemotherapy alone. Interestingly, the interaction between NK cells, which displayed an improved cytotoxicity profile after the combination therapy, and T cells was required to improve the survival, while the presence of B cells deteriorates this effect, as shown in B-cell depleted mice [26, 27]. Also in colorectal cancer, chemotherapy in combination with IL-15 was efficacious. Here, the adjuvant IL-15 potentiates the antitumor activity of 5-fluorouracil alone or in combination with leucovorin [28]. IL-15 induced a significant decrease in chemotherapy-induced gastrointestinal toxicities and reduced the tumor weight in the combination therapy [28].

In all three studies, the statistically significant reduction in tumor growth after combination therapy of chemotherapeutics and IL-15 might be explained by the principle of 'immunogenic cell death' and the increased immune effector functions of $\mathrm{CD} 8^{+} \mathrm{T}$ cells and NK cells. 
Radiofrequency thermal ablation/bone marrow transplantation

Taking into account the chemotherapy-resistance of some tumor cells, not only adjuvant treatments, but also alternative treatments for chemotherapy are being pursued to broaden the treatment options of tumor patients. A possible alternative is the use of radiofrequency thermal ablation (RFA) to kill tumor cells (Figure 2). While the technique is safe and partly effective in solid tumors such as breast cancer [29], the outcome can be enhanced by combining it with immunotherapy as shown in a neu-overexpressing mammary carcinoma mouse model and a 4T1 mouse model [11]. The combination of RFA with the cytokines IL-15 and IL-7, which are both involved in immune stimulation and T-cell survival [30], results in the induction of tumor-specific immune responses in terms of IFN- $\gamma$ secretion, a decreased percentage of myeloid-derived suppressor cells (MDSC) in the spleen and reduced tumor development [11].

However, while RFA can be regarded as an alternative treatment in solid tumors, other alternative or adjuvant treatments are needed for hematological malignancies such as leukemia, lymphoma and myeloma. An established adjuvant treatment is bone marrow transplantation (BMT) (Figure 2). Although the combination of BMT and chemotherapy is effective in obtaining a state of remission, the risk of relapse remains high due to a lack of graft-versus-tumor (GVT) effect [31]. Therefore, additional factors, which avoid the graft-versus-host disease (GVHD) and enhance the GVT effect of BMT, are being investigated. IL-15, given at different time points after BMT, has shown to possess these properties in a leukemic mouse model [32]. The authors suggest that the observed effects can be due to an increase in the percentage of donor-derived NK cells and $\mathrm{CD}^{+}{ }^{\top} \mathrm{T}$ cells, which was further associated with an increase of their antitumor functions. In the early posttransplantation period, NK cells may provide an important defense against infections while specific immunity is still recovering. Therefore, increased NK cell numbers and function due to IL-15 administration after BMT might increase host defense against both infections and the tumor [32]. In addition, these antitumor functions led to a better survival of the IL-15-treated mice after BMT. This survival benefit was also seen when lymphoma bearing mice were treated with BMT followed by IL-15 administration [31].

In conclusion, BMT as well-established adjuvant therapy and RFA as new alternative for chemotherapy are both capable to eradicate the bulk of tumor cells, while IL-15 is able to induce a long-lasting immune response in terms of increasing the number and effector functions of $\mathrm{CD} 8^{+} \mathrm{T}$ cells and NK cells and in reducing the percentage of MDSC.

\section{Other cytokines}

Administration of high-dose IL-2 has been shown to successfully treat tumors in animal models [33], but toxicity associated with this administration [34] and the ability of IL-2 to induce CD4 $4^{+} \mathrm{CD} 25^{+}$ regulatory T-cell activation [12] has compromised its widespread use in the clinic in antitumor therapies. Nowadays, IL-7, IL-12, IL-15 and IL-21 are at the forefront of tumor immunotherapy research, because these cytokines are capable to enhance both NK-cell and T-cell activation and proliferation [6, 34-38] (Figure 2). Combining these cytokines could even have a more potent antitumor effect as revealed in in vivo mouse tumor models. Zeng and colleagues showed that IL-21 cooperates with IL-15 to boost antigen-specific $\mathrm{CD}^{+}{ }^{+}$T-cell numbers and that this cytokine 
combination resulted in a significant regression of B16 melanoma tumor cells as compared to the treatment with each cytokine alone [39]. Also the combination of IL-12, a cytokine favoring naive T cells to differentiate into Thelper (Th)1 cells, and IL-15 results in increased antitumor functions in a B16 melanoma mouse model [40]. In this study, increased levels of activated NK cells (in spleen and lymph nodes) and antigen-specific T cells (in lymph nodes) were found, which may have resulted in the detected tumor regression and survival of the tumor-bearing mice. The synergistic actions of IL-12 and IL-15 could be explained by the reciprocal upregulation of IL-12 and IL-15 receptors by both cytokines [40] and the broad antitumor actions of both cytokines by activating $\mathrm{CD}^{+}{ }^{+}$cells, NK cells, macrophages and granulocytes [40,41]. Finally, the combination of IL-15 and IL-7 has been tested in addition to RFA to increase antitumor effects against mammary tumor cells [11] (vide supra).

\section{Gene therapy approaches}

Unfortunately, the rhIL-15 protein used in clinical trials undergoes a rapid renal clearance and the protein has a short plasma half-life which diminishes its antitumor effects [42]. Furthermore, the systemic administration of IL-15 could increase the risk of potential toxic side effects, including the induction of autoimmunity [6]. Possible solutions to lengthen the time window of IL-15 bioavailability and/or to administer IL-15 locally were found in gene therapy approaches such as transferring the gene(s) of interest, including IL-15, as naked DNA [42-44] or transfecting[45] it into immune or tumor cells through lipofection [41,46, 47], electroporation [45, 48] or viral transduction (by using a lentiviral or adenoviral vector) [49-53]. These approaches are currently being investigated as new methods for IL-15 combination therapies.

Similary as described for cytokine combination therapy (vide supra), tumor-bearing mice treated with irradiated IL-12/IL-15-expressing tumor cells (Figure 2) show a significant tumor rejection in up to $90 \%$ of the mice $[41,46,49,50]$, due to infiltration of $\mathrm{CD}^{+} \mathrm{T}$ cells to the tumor site as shown in depletion experiments [46]. It is currently unclear whether or not NK cells also play a main role in the eradication of tumor cells. Some authors claim that NK cells play only a minor role in tumor rejection after treatment with IL-12/IL-15-expressing tumor cells, because when this combination treatment was given to tumor-bearing NK cell-depleted mice, the tumor was equally diminished in comparison with the treatment of mice where no NK cells were depleted $[41,46]$. Interestingly, when mice were treated with a combination of plasmid-derived IL-15 and IL-21 administered as naked DNA, both cytotoxic CD8 ${ }^{+} T$ cells and NK cells were needed for rejecting the tumor cells, which was ratified in NK cell-depleted lymphoma-bearing [43] and neuroblastoma-bearing [44] mice.

Also in other tumor models like canine transmissible venereal tumors (CTVT), NK cells play an important role in the applied treatment options, i.e. in vivo electroporation of IL- 6 and/or IL-15-expressing plasmids, as demonstrated both in a mouse [45] and a beagle model [48]. The NK-cell activity in these tumors was hampered due to the production of high concentrations of immunosuppressive cytokines, such as transforming growth factor (TGF)- $\beta$, by the tumor cells. Combining a strong antagonist for TGF- $\beta$, e.g. IL-6, and a potent activator of NK cells, e.g. IL-15, resulted in elevated levels of NK cells with a higher cytotoxic capacity, contributing to reduced tumor establishment and growth [45, 48] (Figure 2). Another tumor escape mechanism is the inhibition of 
apoptosis to ensure their development and survival. For this reason, researchers are currently exploring a combination of factors with prominent functions in the programmed cell death pathway together with IL-15. In this context, the combination of caspy2 and IL-15 enhanced both the apoptosis and the immune response induction, leading to (i) a significant reduction in spontaneous lung metastasis in a breast tumor mouse model, (ii) increased survival both in a melanoma and colon tumor mouse model and (iii) the acquisition of a long-time protective immunity against the parental tumor cell rechallenge in a colon tumor mouse model [47] (Figure 2).

Furthermore, linking the coding sequences of the IL-15 protein with a larger protein fragment to increase its molecular weight, and hereby increasing its plasma half-life, is another strategy to increase its efficacy $[42,51,54]$. Therefore, linking IL-15 with the IL-15R $\alpha^{\prime}$ s sushi domain could be a favorable method to enhance the biological antitumor activities of the cytokine (Figure 2). This has been successfully tested in metastatic tumor models, including B16OVA lung metastasis of melanoma, hepatocellular carcinoma and MC38 colon cancer liver metastasis [42, 51]. In addition, combining IL-15 with the $\alpha$-moiety of its receptor is also effective in the treatment of neu-overexpressing tumor-bearing mice [52] (vide infra). Notably, in gene therapies where the gene(s) of interest were administered as naked DNA, it is important to use doses of plasmid within the therapeutic window, because too high concentrations could lead to lymphocytic pneumonitis, while lower concentrations led to higher numbers of activated NK cells and cytotoxic T cells [42].

The activation of NK cells and cytotoxic T cells by IL-15 is only efficient if these effector cells can migrate into the tumor microenvironment to exert their antitumor functions. For this reason, it would be highly interesting to use a chemoattractant, e.g. CCL-21 (Figure 2), to induce the migratory capacity of effector cells into the tumor tissue. Therefore, Zhao and colleagues injected an adenoviral vector expressing both IL-15 and CCL-21 intratumorally in colon cancer-bearing mice, resulting in a decrease of tumor volume as compared with IL-15 or CCL-21 alone [53].

Overall, gene strategies whereby the same cell produces multiple antitumor cytokines or chemokines in a controlled manner (IL-15 in combination with IL-6, IL-12, IL-21 or CCL-21) or whereby the plasma half-life of IL-15 is increased (e.g. IL-15/-15R $\alpha$ fusion constructs), can contribute to reduction in tumor growth of tumor-bearing mice. While the observed antitumor effects can be assigned to increased activation of cytotoxic T cells, the possible involvement of NK cells should not be underestimated.

\section{Antibodies and other targeted therapies}

While IL-15 is able to enhance the cytotoxic activity of $\mathrm{CD}^{+} \mathrm{T}$ cells in vivo, which may result in decreased tumor growth [55], mechanisms which favor tumor survival can counteract these antitumor effects. For example, mutations in an epidermal growth factor receptor (EGFR) may cause overexpression of this receptor and may result in uncontrolled growth of epithelial cells leading to tumors. Indeed, in breast cancer anti-HER2/neu and Cetuximab (Figure 2), both commonly used EGFR inhibitory monoclonal antibodies (moABs), had antitumor effects in mouse models $[56,57]$. These effects were even higher when Cetuximab was combined with IL-15 [56]. Unfortunately, the synergistic effect was not seen after treatment of breast cancer-bearing mice with the anti-Her2/neu 
antibody and IL-15 [57]. The authors suggest that the immune suppressive environment, e.g. programmed cell death protein 1 (PD1) expression on tumor-infiltrating T cells, contributes to the absence of the expected additional or synergistic antitumor effect. Interestingly, Yu P. and colleagues have shown that IL-15, besides its antitumor effects, also enhances the expression of PD1 on $\mathrm{CD}^{+}{ }^{+} \mathrm{T}$ cells and the production of IL-10, due to interaction of CD86 on antigen-presenting cells and cytotoxic T-lymphocyte-associated protein 4 (CTLA-4) on $C D 8^{+} T$ cells. Since both PD1/PDL1 and CD86/CTLA-4 interactions are immune checkpoints during T-cell activation, the activation of $\mathrm{CD} 8^{+} \mathrm{T}$ cells can be suppressed and therefore also the antitumor effects of IL-15. To overcome these immunosuppressive factors, the authors blocked the PD1/PDL1 and CD86/CTLA-4 checkpoints by using an anti-PDL1 and an anti-CTLA-4 antibody (Figure 2) $[58,59]$. When both inhibitory antibodies were used in combination with IL-15, the PD1 expression and IL-10 production were significantly reduced and tumor-bearing mice survived longer after the triple-combination treatment as compared with the IL-15/anti-PDL1 or IL-15/anti-CTLA-4 combination as shown in a metastatic colon carcinoma [59] and a prostate tumor [58] mouse model.

In antitumor treatment, instead of blocking an inhibitory immune checkpoint moABs can also be used to boost the immune system (e.g. anti-CD40, IL-15 fusion proteins; Figure 2). Hereby, the anti-CD40 antibody will bind to CD40 on dendritic cells (DC) for example and will mature the latter. The highly matured DC can in turn present antigens to $\mathrm{CD} 8^{+} \mathrm{T}$ cells, which can eradicate tumor cells. Although the anti-CD40 antibody has got potential as therapeutic antitumor therapy as reported a long time ago [60], the combination of this therapy with IL-15 exerts better antitumor effects as described in different tumor models $[49,61,62]$. These studies show a reduction in tumor growth and an increase in antigen-specific $\mathrm{CD}^{+} \mathrm{T}$-cell infiltration of the tumor after the combination therapy. Moreover, the IL-15/anti-CD40 combination therapy resulted in an elevated NK-cell priming and cytotoxic activity $[61,62]$. Interestingly, also the amount of MDSC and regulatory T cells was diminished in the tumor microenvironment, whereas T helper (Th)1 chemokines were increased as compared with the single treatments [63]. Antitumor immunity might also be boosted by IL-15 fusion proteins, similar as described in the gene therapy section of this paper (vide supra). In this context, IL-15 can be fused to (a part of) IL-15R $\alpha$ alone [18] or to a trifunctional fusion protein consisting of a tumor-specific antibody, IL-15R $\alpha$ and the costimulatory ligand 4-1BBL [64]. Additionally, IL-15 can be mutated by the substitution of one amino acid (N72D) to increase its binding capacities. The mutated IL-15 in combination with the sushi domain of IL-15R $\alpha$ is called ALT803 [65] (Figure 2). The combination of IL-15 with IL-15R $\alpha$ enhances the transpresentation of IL-15, while the trifunctional fusion protein is able to additionally increase the proliferation and activation of (memory) antigen-specific CD8 ${ }^{+} T$ cells, through the interaction of $4-1 \mathrm{BBL}$ with $4-1 \mathrm{BB}$ on $\mathrm{CD} 8^{+} \mathrm{T}$ cells [64]. These constructs were tested in a melanoma mouse model [18, 64], a multiple myeloma mouse model [66] and a bladder cancer rat model [67], resulting in a decreased tumor burden $[18,66,67]$ and a reduction in tumor cell metastases [64].

Recently, everolimus as inhibitor of mammalian target of rapamycin has been thoroughly investigated for its cytotoxic function against multiple tumors (e.g. gastro-entero-pancreatic neuroendocrine tumors, kidney carcinoma, metastatic breast cancer) (Figure 2). Everolimus as stand-alone agent is capable of decreasing tumor size, decreasing Ki-67 expression and increasing rates of apoptosis as shown in a 4T1 tumor mouse model [68]. Zhao et alscrutinized the effect of adding IL-15 as an adjuvant treatment to everolimus. They found that treatment with IL-15 resulted 
in similar antitumor functions with regard to decreased tumor growth and increased apoptosis rates, but there was no synergistic effect when combining IL-15 with everolimus. One possible explanation for this is that the influence of IL-15 on immune cells is balanced by the immunosuppressive effect of everolimus on immune cells, althoughthe underlying mechanisms and interactions should be further examined to fully understand the effect of this combination therapy [68].

Thus, in order to make optimal use of the antitumor effects of IL-15, immune checkpoints or mechanisms that favor tumor survival can be blocked by using inhibitory moABs. In addition, moABs that support the immunostimulatory effects of IL-15 (e.g. anti-CD40) may be used to increase antitumor immunity.

\section{Adoptive T-cell therapy}

Next to blocking immune checkpoints with antibodies and active immune therapies, such as tumor vaccination- or DC-vaccination, also adoptive T-cell therapy is under scrutiny as an antitumor treatment [55, 69] (Figure 2). In antitumor therapy, important factors of a successful adoptive T-cell transfer are on one hand the generation of potently cytotoxic and tumor antigen-specific T cells which can eradicate tumor cells in a direct manner and on the other hand the requirement of the T cells to persist in vivo after adoptive transfer [70]. For this last requirement, it is important that the adoptively transferred T cells obtain memory T-cell characteristics, besides their effector functions. In this perspective, IL-15 has proven to enlarge the memory-properties of adoptively transferred $\mathrm{CD} 8^{+} \mathrm{T}$ cells to effector memory $\mathrm{T}$ cells and central memory $\mathrm{T}$ cells in three weeks and 17 weeks after T-cell transfer, respectively $[55,69]$. Mice treated with IL-15 following adoptive transfer of $\mathrm{CD}^{+} \mathrm{T}$ cells show a survival benefit and tumor regression. Moreover, animals exhibiting complete tumor regression had a significant population of antigen-specific $C D 8^{+} \mathrm{T}$ cells that persisted with IL-15 treatment until 140 days after adoptive transfer and that successfully defended them against tumor rechallenge without IL-15 [69]. Thus, these studies show that IL-15 is capable of increasing the in vivo antitumor functions of (memory) $\mathrm{CD}^{+}{ }^{+} \mathrm{T}$ cells and that these cells can persist in vivo after their adoptive transfer.

\section{Clinical setting}

In vitro and animal experiments with IL-15 have generated a great deal of interest in testing the agent in cancer patients. Immunotherapy experts participating in the US National Cancer Institute Immunotherapy Agent Workshop in 2007 ranked IL-15 as the most important agent to bring to clinical trials. However, as quoted by Dr. Conlon of the $\mathrm{NCl}$, "Researchers have been waiting a long time for clinical grade IL-15, but pharmaceutical companies have largely gotten out of the business of producing new cytokines following some early clinical disappointments with immunotherapy"[71]. For this reason, the $\mathrm{NCl}$ started to produce its own IL-15 to be used in clinical trials. It might also be an explanation why clinical studies where IL-15 has been combined with other antitumor therapies are scarce until now [72]. Nevertheless, three different combination treatments are in the running to date to treat tumor patients (Table 2). In the first combination, chemotherapy (fludarabine/cyclophosphamide) is combined with adoptive cell therapy and recombinant human IL-15 to treat patients with acute myeloid leukemia (NCT01385423), melanoma (NCT01369888) and other advanced solid tumors (NCT01875601). Although this specific combination has not been tested 
in a mouse model, this treatment regimen is promising because adoptive cell therapy in combination with IL-15 or cyclophosphamide in combination with IL-15 were successful to treat rhabdomyosarcoma [26], pulmonary metastases [27], myeloma [69] and melanoma [55] murine models. The second combination strategy being tested in a clinical trial is the use of an IL-15 DC vaccine after surgery in stage III or stage IV melanoma patients (NCT01189383), which will be the first-in-man trial for this combination treatment. The third combination is the use of ALT-803, which is the combination of IL-15 with the sushi domain of IL-15R $\alpha$ and has previously been tested in a murine multiple myeloma and bladder cancer model (vide supra)[66, 67]. The ALT-803 therapy will be used in these clinical trials to treat multiple myeloma (NCT02099539), bladder cancer (NCT02138734) and advanced melanoma (NCT01946789). The clinical studies described here are all ongoing or recently finished, and results have not been published yet. Nevertheless, since IL-15 has proven to be feasible in clinical trials and given its immunostimulatory effects in multiple animal tumor models, more clinical studies on IL-15 combination therapies to treat cancer are expected in the near future.

\section{Discussion}

To summarize, tumor-bearing animals undergoing IL-15 based combinatorial treatment survived for a longer time period and showed a significant reduction in tumor size and growth. In two studies only, the IL-15 combination therapy (anti-HER2 + IL-15 or everolimus + IL-15) did not display any synergistic effects, probably due to the presence of MDSC and PD1 on tumor-infiltrating T cells [57] or due to the immunosuppressive effects of everolimus itself [68]. In these cases, extra immunotherapeutic strategies such as anti-PD1/PDL1 antibodies might be required to bypass the immunosuppressive effects.

In addition to the tumor suppressive environment, the short IL-15 half-life can impede its in vivo antitumor effects. To increase this half-life , IL-15 can be complexed with the (sushi domain of the) $\alpha$-moiety of its receptor $[18,42,64]$. Using this strategy, not only the molecular weight of IL-15 is increased to elevate its half-life, but IL-15 will also be better (trans)presented to the IL-15 $\beta \gamma$ receptor which increases IL-15 signaling $[16,73]$. Since this pre-complexation has led to increased antitumor effects in mouse models $[18,42,64]$, it is very interesting to test this complex in clinical trials to treat tumor patients. To date, there are three clinical studies exploiting the IL-15:IL-15R $\alpha$ pre-complexation principle to treat patients with multiple myeloma, advanced melanoma and bladder cancer.

Regardless of the type of IL-15 combination therapy, in order to better understand the antitumor functions of IL-15, it will be important to know which cells are responsible for the observed effects. IL-15 has shown to be a stimulator both of $\mathrm{CD}^{+}$T cells of the adaptive immune system and NK cells of the innate immune system as described in many in vitro studies [6]. In the in vivo setting, IL-15 is able to enhance the activity of tumor-reactive $\operatorname{CD} 8^{+} T$ cells $[55,74]$, but it remains to be established whether NK cells are also key players in the IL-15-mediated antitumor immunity. In general, most studies reported an enhanced percentage and/or cytotoxic activity of NK cells [18, 27, 32, 39, 40, 42, $43,45,48,51,56,61,62]$. Moreover, four of these studies indicated the indispensability of NK cells 
to improve in vivo antitumor immunity, since NK-cell depletion resulted in dampening or even abolishment of the antitumor effect $[43,45,48,51]$. On the contrary, Di Carlo and colleagues showed in a murine small cell lung cancer model that NK cells are not required for antitumor functions, because NK cell-depleted mice demonstrated a similar antitumor effect as wild-type tumor bearing-mice after treatment with the IL-12/IL-15 combination therapy [41]. The authors suggest that in NK-cell depleted mice the combination therapy mainly targets macrophages and granulocytes to conduct the antitumor functions, indicating the importance of this combination therapy in MHC class I-negative tumors. Overall, in the antitumor setting it is certainly interesting to investigate the involvement of NK cells and other cell types of the innate and adaptive immune system besides $\mathrm{CD}^{+} \mathrm{T}$ cells.

In conclusion, the most promising antitumor IL-15 combination therapy must tackle the most burning current hurdles, being (i) tumor burden, (ii) the tumor immunosuppressive environment and (iii) the short half-life of the cytokine. In this perspective, an ideal antitumor therapy could be the combination of (1) chemotherapy or surgery to decrease the tumor burden; (2) anti-PD1 and anti-CTLA-4 antibodies to block immune inhibitory molecules; and (3) pre-complexed IL-15:IL-15R $\alpha$ fusion proteins to increase the half-life and signaling of IL-15.

\section{Conflict of interest}

The authors declare no conflict of interest.

\section{Acknowledgments}

This work is supported by the research grant STK2012-193 of the Belgian Stichting tegen Kanker. J. Van den Bergh receives a fellowship from the University of Antwerp. The authors like to thank B. Van den Bergh for his contribution to Figure 1 and 2.

\section{References}

[1] Cheever MA. Twelve immunotherapy drugs that could cure cancers. Immunol Rev. 2008;222:35768.

[2] Carson WE, Giri JG, Lindemann MJ, Linett ML, Ahdieh M, Paxton R, et al. Interleukin (IL) 15 is a novel cytokine that activates human natural killer cells via components of the IL-2 receptor. J Exp Med. 1994;180:1395-403.

[3] Lewko WM, Smith TL, Bowman DJ, Good RW, Oldham RK. Interleukin-15 and the growth of tumor derived activated T-cells. Cancer Biother. 1995;10:13-20.

[4] Armitage RJ, Macduff BM, Eisenman J, Paxton R, Grabstein KH. IL-15 has stimulatory activity for the induction of B cell proliferation and differentiation. J Immunol. 1995;154:483-90.

[5] Chen J, Niu H, He W, Ba D. Antitumor activity of expanded human tumor-infiltrating gammadelta T lymphocytes. Int Arch Allergy Immunol. 2001;125:256-63. 
[6] Jakobisiak M, Golab J, Lasek W. Interleukin 15 as a promising candidate for tumor immunotherapy. Cytokine Growth Factor Rev. 2011;22:99-108.

[7] Croce M, Orengo AM, Azzarone B, Ferrini S. Immunotherapeutic applications of IL-15. Immunotherapy. 2012;4:957-69.

[8] Fehniger TA, Cooper MA, Caligiuri MA. Interleukin-2 and interleukin-15: immunotherapy for cancer. Cytokine Growth Factor Rev. 2002;13:169-83.

[9] Kennedy MK, Park LS. Characterization of interleukin-15 (IL-15) and the IL-15 receptor complex. J Clin Immunol. 1996;16:134-43.

[10] Waldmann T, Tagaya Y, Bamford R. Interleukin-2, interleukin-15, and their receptors. Int Rev Immunol. 1998;16:205-26.

[11] Habibi M, Kmieciak M, Graham L, Morales JK, Bear HD, Manjili MH. Radiofrequency thermal ablation of breast tumors combined with intralesional administration of IL-7 and IL-15 augments anti-tumor immune responses and inhibits tumor development and metastasis. Breast Cancer Res Treat. 2009;114:423-31.

[12] Nelson BH. IL-2, regulatory T cells, and tolerance. J Immunol. 2004;172:3983-8.

[13] Burkett PR, Koka R, Chien M, Chai S, Boone DL, Ma A. Coordinate expression and trans presentation of interleukin (IL)-15Ralpha and IL-15 supports natural killer cell and memory CD8+ T cell homeostasis. J Exp Med. 2004;200:825-34.

[14] Kobayashi H, Dubois S, Sato N, Sabzevari H, Sakai Y, Waldmann TA, et al. Role of trans-cellular IL15 presentation in the activation of NK cell-mediated killing, which leads to enhanced tumor immunosurveillance. Blood. 2005;105:721-7.

[15] Schluns KS, Stoklasek T, Lefrancois L. The roles of interleukin-15 receptor alpha: transpresentation, receptor component, or both? Int J Biochem Cell Biol. 2005;37:1567-71.

[16] Lodolce JP, Burkett PR, Koka RM, Boone DL, Ma A. Regulation of lymphoid homeostasis by interleukin-15. Cytokine Growth Factor Rev. 2002;13:429-39.

[17] Rubinstein MP, Kovar M, Purton JF, Cho JH, Boyman O, Surh CD, et al. Converting IL-15 to a superagonist by binding to soluble IL-15R\{alpha\}. Proc Natl Acad Sci U S A. 2006;103:9166-71.

[18] Stoklasek TA, Schluns KS, Lefrancois L. Combined IL-15/IL-15Ralpha immunotherapy maximizes IL-15 activity in vivo. J Immunol. 2006;177:6072-80.

[19] Madan RA, Schwaab T, Gulley JL. Strategies for optimizing the clinical impact of immunotherapeutic agents such as sipuleucel-T in prostate cancer. J Natl Compr Canc Netw. 2012;10:1505-12.

[20] Kepp O, Galluzzi L, Martins I, Schlemmer F, Adjemian S, Michaud M, et al. Molecular determinants of immunogenic cell death elicited by anticancer chemotherapy. Cancer Metastasis Rev. 2011;30:61-9.

[21] Martins I, Tesniere A, Kepp O, Michaud M, Schlemmer F, Senovilla L, et al. Chemotherapy induces ATP release from tumor cells. Cell Cycle. 2009;8:3723-8.

[22] Vacchelli E, Galluzzi L, Fridman WH, Galon J, Sautes-Fridman C, Tartour E, et al. Trial watch: Chemotherapy with immunogenic cell death inducers. Oncoimmunology. 2012;1:179-88.

[23] Vacchelli E, Aranda F, Eggermont A, Galon J, Sautes-Fridman C, Cremer I, et al. Trial Watch: Chemotherapy with immunogenic cell death inducers. Oncoimmunology. 2014;3:e27878.

[24] Gelbard A, Garnett CT, Abrams SI, Patel V, Gutkind JS, Palena C, et al. Combination chemotherapy and radiation of human squamous cell carcinoma of the head and neck augments CTL-mediated lysis. Clin Cancer Res. 2006;12:1897-905.

[25] Hodge JW, Garnett CT, Farsaci B, Palena C, Tsang KY, Ferrone S, et al. Chemotherapy-induced immunogenic modulation of tumor cells enhances killing by cytotoxic $T$ lymphocytes and is distinct from immunogenic cell death. Int J Cancer. 2013;133:624-36.

[26] Evans R, Fuller JA, Christianson G, Krupke DM, Troutt AB. IL-15 mediates anti-tumor effects after cyclophosphamide injection of tumor-bearing mice and enhances adoptive immunotherapy: the potential role of NK cell subpopulations. Cell Immunol. 1997;179:66-73. 
[27] Chapoval Al, Fuller JA, Kremlev SG, Kamdar SJ, Evans R. Combination chemotherapy and IL-15 administration induce permanent tumor regression in a mouse lung tumor model: NK and $T$ cell-mediated effects antagonized by B cells. J Immunol. 1998;161:6977-84.

[28] Cao S, Troutt AB, Rustum YM. Interleukin 15 protects against toxicity and potentiates antitumor activity of 5 -fluorouracil alone and in combination with leucovorin in rats bearing colorectal cancer. Cancer Res. 1998;58:1695-9.

[29] Vogl TJ, Farshid P, Naguib NN, Zangos S. Thermal ablation therapies in patients with breast cancer liver metastases: a review. Eur Radiol. 2013;23:797-804.

[30] Kim HR, Hwang KA, Park SH, Kang I. IL-7 and IL-15: biology and roles in T-Cell immunity in health and disease. Crit Rev Immunol. 2008;28:325-39.

[31] Katsanis E, XU Z, Panoskaltsis-Mortari A, Weisdorf DJ, Widmer MB, Blazar BR. IL-15 administration following syngeneic bone marrow transplantation prolongs survival of lymphoma bearing mice. Transplantation. 1996;62:872-5.

[32] Alpdogan O, Eng JM, Muriglan SJ, Willis LM, Hubbard VM, Tjoe KH, et al. Interleukin-15 enhances immune reconstitution after allogeneic bone marrow transplantation. Blood. 2005; 105:865-73.

[33] Hancock BW, Rees RC. Interleukin-2 and cancer therapy. Cancer Cells. 1990;2:29-32.

[34] Wang G, Tschoi M, Spolski R, Lou $Y$, Ozaki K, Feng C, et al. In vivo antitumor activity of interleukin 21 mediated by natural killer cells. Cancer Res. 2003;63:9016-22.

[35] Bhave NS, Carson WE, 3rd. Immune modulation with interleukin-21. Ann N Y Acad Sci. 2009;1182:39-46.

[36] Brunda MJ. Interleukin-12. J Leukoc Biol. 1994;55:280-8.

[37] Li Y, Bleakley M, Yee C. IL-21 influences the frequency, phenotype, and affinity of the antigenspecific CD8 T cell response. J Immunol. 2005;175:2261-9.

[38] Li Y, Liu S, Hernandez J, Vence L, Hwu P, Radvanyi L. MART-1-specific melanoma tumorinfiltrating lymphocytes maintaining CD28 expression have improved survival and expansion capability following antigenic restimulation in vitro. J Immunol. 2010;184:452-65.

[39] Zeng R, Spolski R, Finkelstein SE, Oh S, Kovanen PE, Hinrichs CS, et al. Synergy of IL-21 and IL-15 in regulating CD8+ T cell expansion and function. J Exp Med. 2005;201:139-48.

[40] Lasek W, Golab J, Maslinski W, Switaj T, Balkowiec EZ, Stoklosa T, et al. Subtherapeutic doses of interleukin-15 augment the antitumor effect of interleukin-12 in a B16F10 melanoma model in mice. Eur Cytokine Netw. 1999;10:345-56.

[41] Di Carlo E, Comes A, Basso S, De Ambrosis A, Meazza R, Musiani P, et al. The combined action of IL-15 and IL-12 gene transfer can induce tumor cell rejection without T and NK cell involvement. J Immunol. 2000;165:3111-8.

[42] Ochoa MC, Fioravanti J, Rodriguez I, Hervas-Stubbs S, Azpilikueta A, Mazzolini G, et al. Antitumor immunotherapeutic and toxic properties of an HDL-conjugated chimeric IL-15 fusion protein. Cancer Res. 2013;73:139-49.

[43] Kishida T, Asada H, Itokawa Y, Cui FD, Shin-Ya M, Gojo S, et al. Interleukin (IL)-21 and IL-15 genetic transfer synergistically augments therapeutic antitumor immunity and promotes regression of metastatic lymphoma. Mol Ther. 2003;8:552-8.

[44] Kowalczyk A, Wierzbicki A, Gil M, Bambach B, Kaneko Y, Rokita H, et al. Induction of protective immune responses against NXS2 neuroblastoma challenge in mice by immunotherapy with GD2 mimotope vaccine and IL-15 and IL-21 gene delivery. Cancer Immunol Immunother. 2007;56:1443-58.

[45] Lin CY, Chuang TF, Liao KW, Huang YJ, Pai CC, Chu RM. Combined immunogene therapy of IL-6 and IL-15 enhances anti-tumor activity through augmented NK cytotoxicity. Cancer Lett. 2008;272:285-95.

[46] Comes A, Di Carlo E, Musiani P, Rosso O, Meazza R, Chiodoni C, et al. IFN-gamma-independent synergistic effects of IL-12 and IL-15 induce anti-tumor immune responses in syngeneic mice. Eur J Immunol. 2002;32:1914-23. 
[47] Yang Y, Zhang XM, Zhang N, Cheng L, Li C, Zhang S, et al. IL15 combined with Caspy2 provides enhanced therapeutic efficiency against murine malignant neoplasm growth and metastasis. Cancer Gene Ther. 2012;19:460-7.

[48] Chou PC, Chuang TF, Jan TR, Gion HC, Huang YC, Lei HJ, et al. Effects of immunotherapy of IL-6 and IL-15 plasmids on transmissible venereal tumor in beagles. Vet Immunol Immunopathol. 2009;130:25-34.

[49] Lasek W, Basak G, Switaj T, Jakubowska AB, Wysocki PJ, Mackiewicz A, et al. Complete tumour regressions induced by vaccination with IL-12 gene-transduced tumour cells in combination with IL-15 in a melanoma model in mice. Cancer Immunol Immunother. 2004;53:363-72.

[50] Basak GW, Zapala L, Wysocki PJ, Mackiewicz A, Jakobisiak M, Lasek W. Interleukin 15 augments antitumor activity of cytokine gene-modified melanoma cell vaccines in a murine model. Oncol Rep. 2008;19:1173-9.

[51] Chang CM, Lo CH, Shih YM, Chen Y, Wu PY, Tsuneyama K, et al. Treatment of hepatocellular carcinoma with adeno-associated virus encoding interleukin-15 superagonist. Hum Gene Ther. 2010;21:611-21.

[52] Steel JC, Ramlogan CA, Yu P, Sakai Y, Forni G, Waldmann TA, et al. Interleukin-15 and its receptor augment dendritic cell vaccination against the neu oncogene through the induction of antibodies partially independent of CD4 help. Cancer Res. 2010;70:1072-81.

[53] Zhao DX, Li ZJ, Zhang Y, Zhang XN, Zhao KC, Li YG, et al. Enhanced antitumor immunity is elicited by adenovirus-mediated gene transfer of CCL21 and IL-15 in murine colon carcinomas. Cell Immunol. 2014;289:155-61.

[54] Kaspar M, Trachsel E, Neri D. The antibody-mediated targeted delivery of interleukin-15 and GM-CSF to the tumor neovasculature inhibits tumor growth and metastasis. Cancer Res. 2007;67:4940-8.

[55] Klebanoff CA, Finkelstein SE, Surman DR, Lichtman MK, Gattinoni L, Theoret MR, et al. IL-15 enhances the in vivo antitumor activity of tumor-reactive CD8+ T cells. Proc Natl Acad Sci U S A. 2004;101:1969-74.

[56] Roberti MP, Rocca YS, Amat M, Pampena MB, Loza J, Colo F, et al. IL-2- or IL-15-activated NK cells enhance Cetuximab-mediated activity against triple-negative breast cancer in xenografts and in breast cancer patients. Breast Cancer Res Treat. 2012;136:659-71.

[57] Xu M, Du X, Liu M, Li S, Li X, Fu YX, et al. The tumor immunosuppressive microenvironment impairs the therapy of anti-HER2/neu antibody. Protein Cell. 2012;3:441-9.

[58] Yu P, Steel JC, Zhang M, Morris JC, Waitz R, Fasso M, et al. Simultaneous inhibition of two regulatory T-cell subsets enhanced Interleukin-15 efficacy in a prostate tumor model. Proc Natl Acad Sci U S A. 2012;109:6187-92.

[59] Yu P, Steel JC, Zhang M, Morris JC, Waldmann TA. Simultaneous blockade of multiple immune system inhibitory checkpoints enhances antitumor activity mediated by interleukin-15 in a murine metastatic colon carcinoma model. Clin Cancer Res. 2010;16:6019-28.

[60] Donepudi M, Quach DD, Mokyr MB. Signaling through CD40 enhances cytotoxic T lymphocyte generation by $\mathrm{CD} 8+\mathrm{T}$ cells from mice bearing large tumors. Cancer Immunol Immunother. 1999;48:153-64.

[61] Zhang M, Yao Z, Dubois S, Ju W, Muller JR, Waldmann TA. Interleukin-15 combined with an antiCD40 antibody provides enhanced therapeutic efficacy for murine models of colon cancer. Proc Natl Acad Sci U S A. 2009;106:7513-8.

[62] Zhang M, Ju W, Yao Z, Yu P, Wei BR, Simpson RM, et al. Augmented IL-15Ralpha expression by CD40 activation is critical in synergistic CD8 T cell-mediated antitumor activity of anti-CD40 antibody with IL-15 in TRAMP-C2 tumors in mice. J Immunol. 2012;188:6156-64.

[63] Weiss JM, Back TC, Scarzello AJ, Subleski JJ, Hall VL, Stauffer JK, et al. Successful immunotherapy with IL-2/anti-CD40 induces the chemokine-mediated mitigation of an immunosuppressive tumor microenvironment. Proc Natl Acad Sci U S A. 2009;106:19455-60. 
[64] Kermer V, Hornig N, Harder M, Bondarieva A, Kontermann RE, Muller D. Combining antibodydirected presentation of $\mathrm{IL}-15$ and $4-1 \mathrm{BBL}$ in a trifunctional fusion protein for cancer immunotherapy. Mol Cancer Ther. 2014;13:112-21.

[65] Zhu X, Marcus WD, Xu W, Lee HI, Han K, Egan JO, et al. Novel human interleukin-15 agonists. J Immunol. 2009;183:3598-607.

[66] Xu W, Jones M, Liu B, Zhu X, Johnson CB, Edwards AC, et al. Efficacy and mechanism-of-action of a novel superagonist interleukin-15: interleukin-15 receptor alphaSu/Fc fusion complex in syngeneic murine models of multiple myeloma. Cancer Res. 2013;73:3075-86.

[67] Gomes-Giacoia E, Miyake M, Goodison S, Sriharan A, Zhang G, You L, et al. Intravesical ALT-803 and BCG treatment reduces tumor burden in a carcinogen induced bladder cancer rat model; a role for cytokine production and NK cell expansion. PLoS One. 2014;9:e96705.

[68] Zhao N, Li X, He X, Qiu Y, Zhu L, Qi F. Interleukin-15 gene therapy and the mammalian target of rapamycin inhibitor everolimus inhibit the growth of metastatic breast cancer. J Gene Med. 2013;15:366-74.

[69] Roychowdhury S, May KF, Jr., Tzou KS, Lin T, Bhatt D, Freud AG, et al. Failed adoptive immunotherapy with tumor-specific T cells: reversal with low-dose interleukin 15 but not lowdose interleukin 2. Cancer Res. 2004;64:8062-7.

[70] Riddell SR. Engineering antitumor immunity by T-cell adoptive immunotherapy. Hematology Am Soc Hematol Educ Program. 2007:250-6.

[71] Waldmann T CK. http://www.cancer.gov/ncicancerbulletin/012412/page8. January 21th, 2012

[72] NIH. https://clinicaltrials.gov/. June, 2013

[73] Stonier SW, Schluns KS. Trans-presentation: a novel mechanism regulating IL-15 delivery and responses. Immunol Lett. 2010;127:85-92.

[74] Teague RM, Sather BD, Sacks JA, Huang MZ, Dossett ML, Morimoto J, et al. Interleukin-15 rescues tolerant $\mathrm{CD} 8+\mathrm{T}$ cells for use in adoptive immunotherapy of established tumors. Nat Med. 2006;12:335-41. 
Figure legends

Figure 1. Mechanisms whereby IL-15 can bind to its effector cells. IL-15 can be presented by the IL$15 R \alpha$-moiety (green) to the IL-15R $\beta \gamma$-subuntis (purple) in a membrane-bound state through a process called transpresentation (1) or in a soluble state (2). IL-15 can also bind the IL-15R $\beta$ Ysubunits directly, but with a lower affinity (3).

Figure 2. IL-15 in combination with (1) chemotherapy/Everolimus/ACT/BMT/RFA, (2) IL-21, (3) IL-12, (4) IL-6, (5) Cetuximab or anti-HER2, (6) anti-PDL1, (7) anti-CD40, (8) anti-CTLA-4, (9) IL15R $\alpha / A L T 803,(10)$ IL-15R $\alpha$ and 4-1BBL, (11) Caspy 2 and (12) CCL-21. Interactions between CTL and APC: (a) CD40-CD40L, (b) PD1-PDL1, (c) TCR-MHC I and (d) CTLA-4-CD80/CD86. Abbreviations. ACT, Adoptive cell transfer; APC, antigen-presenting cell; BMT, Bone marrow transplantation; CTL, Cytotoxic T lymphocyte; IL, Interleukin; NK, Natural Killer; RFA, Radiofrequency thermal ablation; TGF- $\beta$, tumor growth factor; Th, T Helper. 


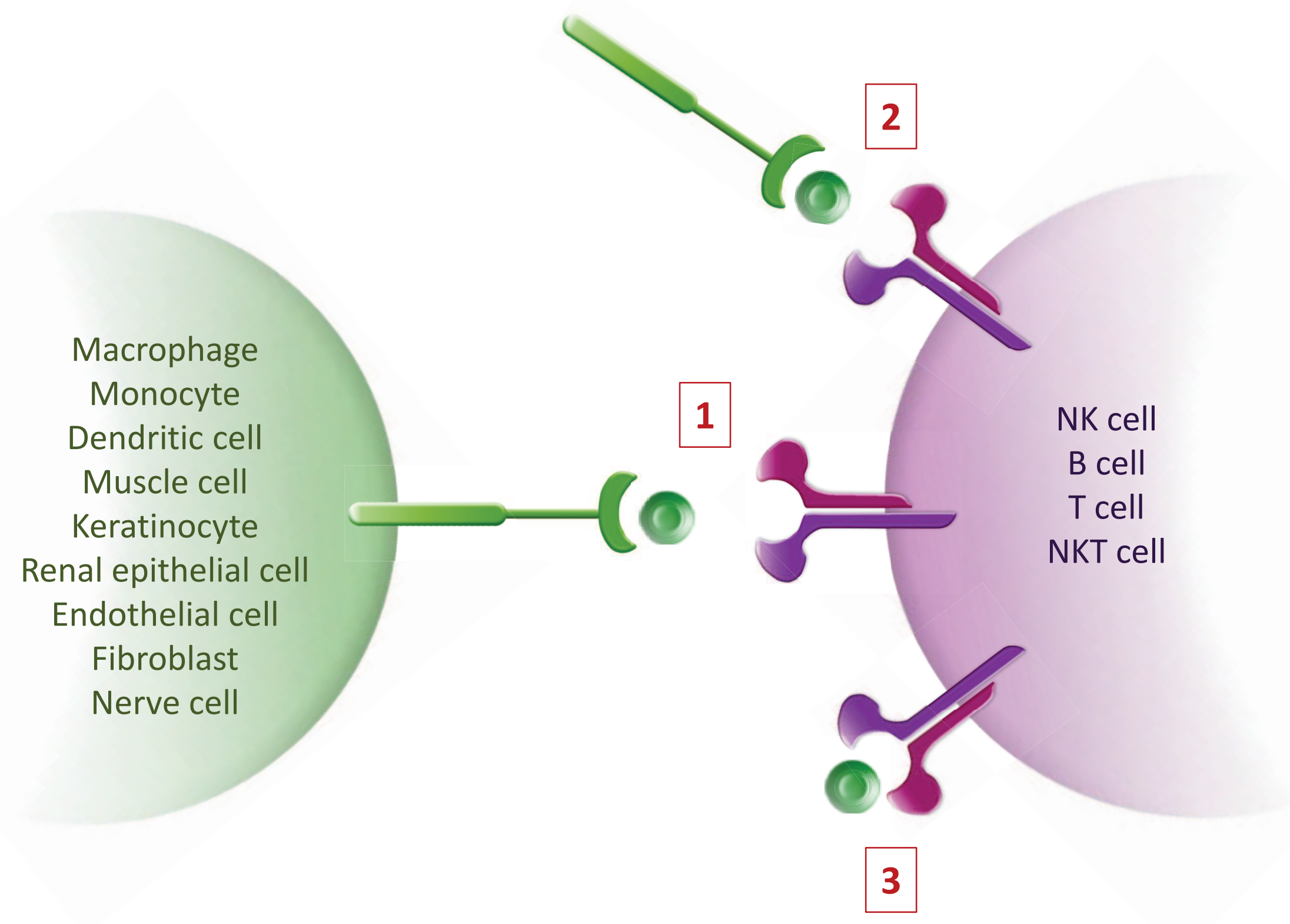

Figure 1. Mechanisms whereby IL-15 can bind to its effector cells. IL-15 can be presented by the IL-15R $\alpha$-moiety (green) to the IL-15R $\beta$ subuntis (purple) in a membrane-bound state through a process called transpresentation (1) or in a soluble state (2). IL-15 can also bind the IL-15RßY-subunits directly, but with a lower affinity (3). 


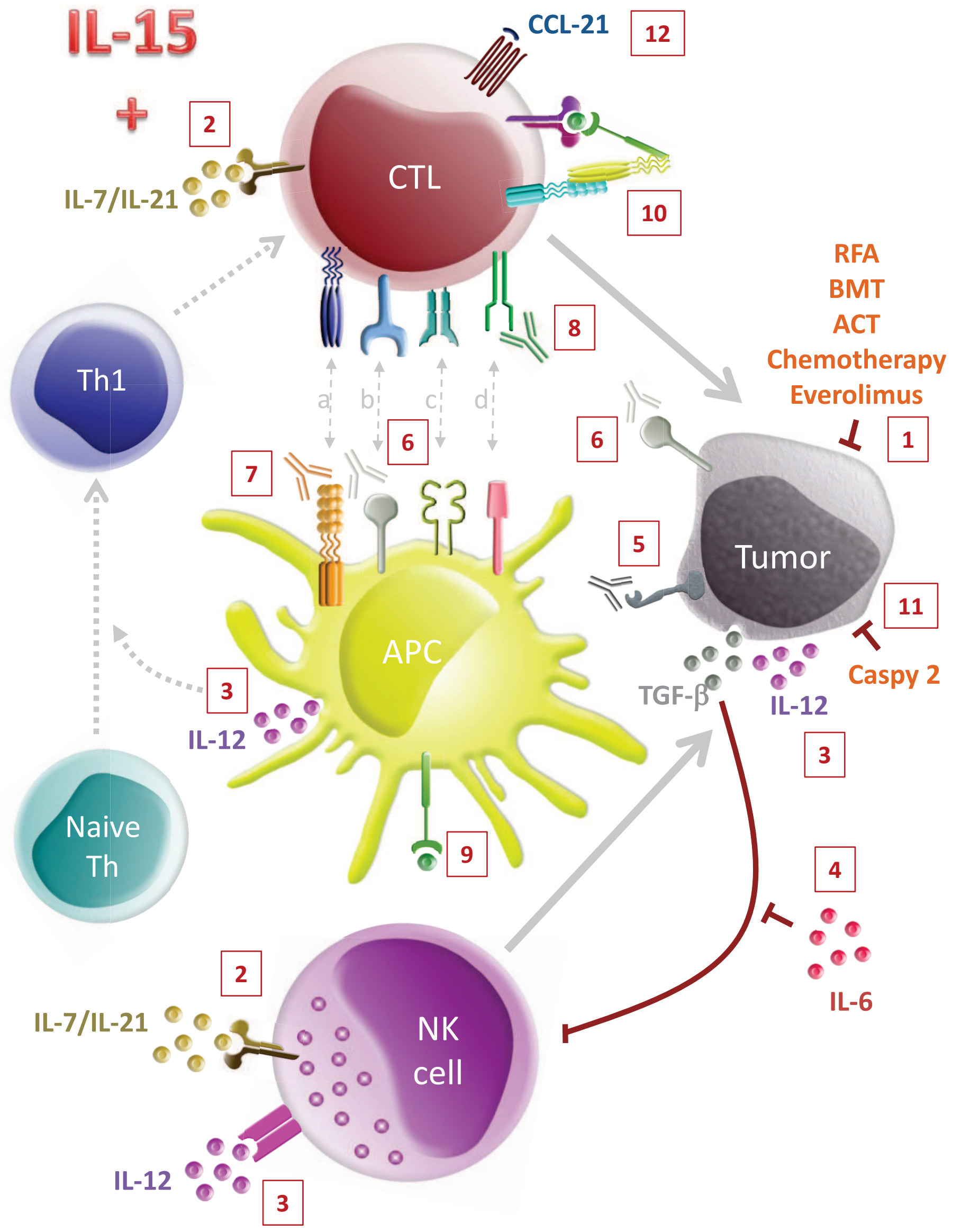

Figure 2. IL-15 in combination with (1) chemotherapy/Everolimus/ACT/BMT/RFA, (2) IL-21, (3) IL-12, (4) IL-6, (5) Cetuximab or anti-HER2, (6) anti-PDL1, (7) anti-CD40, (8) anti-CTLA-4, (9) IL-15R $\alpha / A L T 803,(10)$ IL-15R $\alpha$ and 4-1BBL, (11) Caspy 2 and (12) CCL-21. Interactions between CTL and APC: (a) CD40-CD40L, (b) PD1-PDL1, (c) TCR-MHC I and (d) CTLA-4-CD80/CD86. Abbreviations. ACT, Adoptive cell transfer; APC, antigen-presenting cell; BMT, Bone marrow transplantation; CTL, Cytotoxic T lymphocyte; IL, Interleukin; NK, Natural Killer; RFA, Radiofrequency thermal ablation; TGF- $\beta$, tumor growth factor; Th, T Helper. 